\title{
PATTERN FORMATION IN NOISY SELF-REPLICATING SPOTS
}

\author{
Andreea Munteanu ${ }^{1, *}$ and Ricard V. Solé ${ }^{1,2}$ \\ ${ }^{1}$ ICREA-Complex Systems Lab, Universitat Pompeu Fabra (GRIB), Dr Aiguader 80, 08003 Barcelona, Spain \\ ${ }^{2}$ Santa Fe Institute, 1399 Hyde Park Road, Santa Fe NM 87501, USA
}

\begin{abstract}
Among reaction-diffusion systems showing Turing patterns, the diffusive Gray-Scott model [Pearson, J. A., 1993 , Science 261, 189] stands out by showing self-replicating patterns (spots), which makes it the ideal simple model for developmental research. A first study of the influence of noise in the Gray-Scott model was performed by Lesmes et al. [2003 Phys. Rev. Lett. 91, 238301] concluding that there is an optimal noise intensity for which spot multiplication is maximal. Here we show in details the transition from non-spotlike to spotlike pattern, with the identification of a wide range of noise intensities instead of an optimal value for which this transition occurs, suggesting a very robust intrinsicly noise-based emergent property.
\end{abstract}

Keywords: Turing patterns, reaction-diffusion, noise, self-replication

The major step forward in the modern theory of pattern formation was given by Turing [1952], who used the linear analysis to determine the conditions necessary for the creation of spatial patterns in two-component reaction-diffusion systems. More recently, Gierer and Meinhardt [2000], Koch and Meinhardt [1994] and independently Segel and Jackson [1972] postulated that the interplay between two antagonic feedbacks is essential for pattern formation. On one hand, the positive feedback should consist in the self-enhancement or autocatalysis of one of the chemical components - generally called activator -, a reaction necessary for small perturbations to be amplified. On the other hand, the increase in activator's concentration must be complemented by a fast-diffusing response in order to obtain pattern formation. According to the nature of the antagonic response, the resulting systems are either of activator-inhibitor type - an inhibitory substance is produced by the activator that slows down the latter's production, or of activator-substrate type - a substrate substance is consumed during autocatalysis leading to a depletion of the activator and the relaxation of the self-enhancement.

The most studied examples of the two types of reactiondiffusion systems are the Meinhardt system [Gierer and Meinhardt 2000] and the diffusive Gray-Scott system [Pearson 1993], respectively. The complex interplay between activator and inhibitor or substrate chemical, aided by the reaction and diffusion components create most startling spatiotemporal patterns, such as spots, stripes, travelling waves, spot replication, and spatio-temporal chaos, in a nutshell, a clear example of Turing patterns. The Turing patterns are characterized by the active role that diffusion plays in destabilizing the homogeneous steady state of the system. They emerge spontaneously as the system is driven into a state where it is unstable towards the growth of finite-wavelength stationary perturbations. Interesting enough, the replication characteristic is a particularity of the diffusive Gray-Scott model alone, which makes it the ideal model for the unfolding development of a proto-organism. In this case, cell-like localized structures

*Corresponding author: E-mail: andreea.munteanu@upf.edu; Phone: +34 935422834; Fax: +34 932213237 grow, deform and make replica of themselves until they occupy the entire space.

The Turing patterns from the work of Pearson [1993] on the diffusive Gray-Scott model were confirmed experimentally by Lee et al. [1993], including the spot replication [Lee et al. 1994]. Theoretically, extensive work exist in the literature on the dynamics of this model concerning the "spot replication" in one, two and three dimensions [Muratov and Osipov 2000]. Recently, a first study of the influence of noise in the GrayScott model was performed by Lesmes et al. [2003] concluding that there is an optimal noise intensity for which spot multiplication is maximal. Here we show in details the transition from non-spotlike to spotlike pattern, with the identification of a wide range of noise intensities for which this transition occurs, suggesting a very robust intrinsicly noise-based emergent property.

\section{The Model}

The model was originally introduced in Gray and Scott [1985] as an isothermal system with chemical feedback in a continuously fed, well-stirred tank reactor, where the last property implied the lack of diffusion. The analysis of the system revealed stationary states, sustained oscillations and even chaotic behavior. The model considers the chemical reactions

$$
\begin{aligned}
U+2 V & \rightarrow U+3 V \\
V & \rightarrow P,
\end{aligned}
$$

describing the autocatalytic growth of an activator $V$ on the continuously fed substrate, $U$ and the decay of the former in the inert product $P$, subsequently removed from the system. A major development was performed by Pearson [1993] who introduced the role of space by relaxing the constraint of a well-stirred tank and studied the system in two dimensions, in the limit of small diffusion. In two dimensions, the concentrations of the two chemical components, $u(x, y, t)$ and $v(x, y, t)$ are given by 
(a)

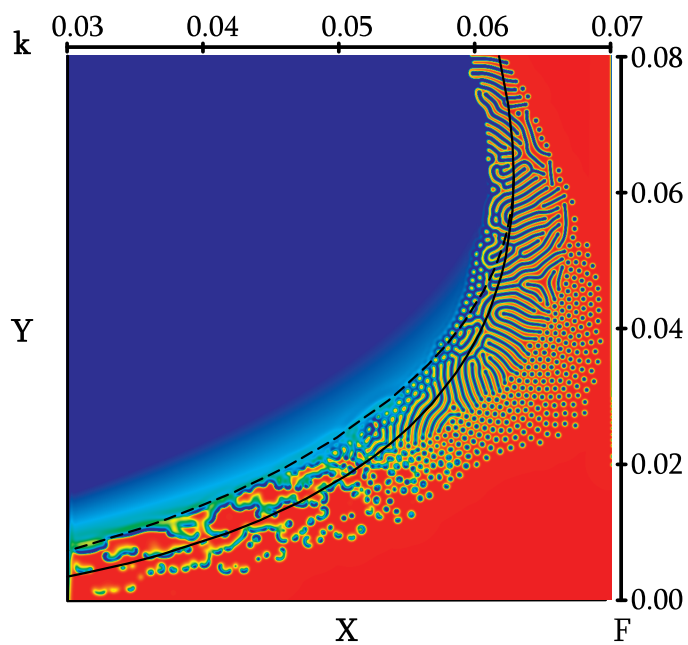

(b)



(e)

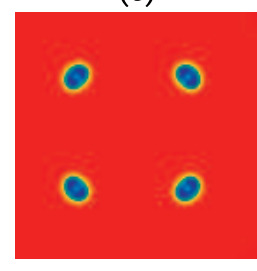

(c)

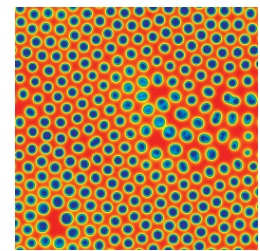

(f)

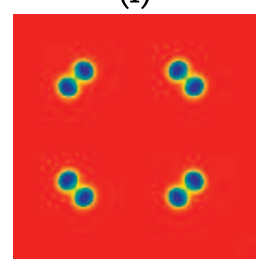

(d)

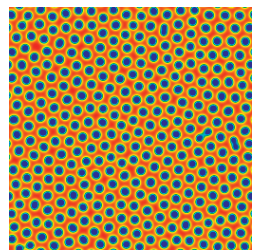

(g)

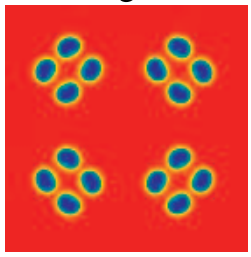

FIG. 1 Pearson model. (a) Illustrative static version of the final patterns produced by the system. The bifurcation curves are drawn: the saddle-node (solid line) and the Hopf bifurcation curve (dashed line). The spot-multiplication patterns as classified in Pearson [1993]: the (b) $\epsilon$, (c) $\zeta$ and (d) $\lambda$ patterns. Panels (e), (f) and (g) illustrate the spot-multiplication process.

$$
\begin{aligned}
& \frac{\partial u}{\partial t}=D_{u} \nabla^{2} u-u v^{2}+F(1-u) \\
& \frac{\partial v}{\partial t}=D_{v} \nabla^{2} v+u v^{2}-(F+k) v
\end{aligned}
$$

where $D_{u}$ and $D_{v}$ are the diffusion coefficients, $F$ is the dimensionless flow rate (the inverse of the residence time) and $k$ is decay constant of the activator, $V$. The original study involved fixed diffusion coefficients, $D_{u}=2 \times 10^{-5}$ and $D_{v}=10^{-5}$, with $F$ and $k$ being the control parameters.

As a typical Turing pattern, the system has a steady state stable with respect to homogeneous temporal oscillations which becomes unstable towards standing, space-periodic perturbations when diffusion is taken into account [see Mazin et al. 1996, for a detailed linear analysis of the Gray-Scott model]. The linear analysis shows the existence of a trivial homogeneous steady state $\left(u_{\mathrm{R}}, v_{\mathrm{R}}\right)=(1,0)$ - referred to as the red stage - which reflects the continuously supplied substrate and is stable for any pair of $(F, k)$. For certain values of $F$ and $k$, namely when $1-4 \delta^{2} F \geq 0$, other two steady states exist, $\left(u_{\mathrm{B}}, v_{\mathrm{B}}\right)$ and $\left(u_{\mathrm{I}}, v_{\mathrm{I}}\right)$ :

$$
\begin{aligned}
& u_{\mathrm{B}, \mathrm{I}}=\frac{1}{2}\left(1 \pm \sqrt{1-4 \delta^{2} F}\right), \\
& v_{\mathrm{B}, \mathrm{I}}=\frac{1}{2 \delta}\left(1 \mp \sqrt{1-4 \delta^{2} F}\right) .
\end{aligned}
$$

When these two steady states exist, it results from Eqs. (5)-(6) that $\left(u_{\mathrm{I}}, v_{\mathrm{I}}\right)$ is always unstable, while $\left(u_{\mathrm{B}}, v_{\mathrm{B}}\right)$ - the blue state - may be either stable or unstable, with the stability conditions consisting in

$$
\begin{array}{r}
\operatorname{Tr}(A)=-v_{0}^{2}+k<0 \\
|A|=\left(v_{0}{ }^{2}-F\right)(F+k)>0 .
\end{array}
$$

More precisely, for the values of $(F, k)$ for which $\operatorname{Tr}^{2}(A)-$ $4|A|<0$, the blue state crosses a Hopf bifurcation [Strogatz 1994] when the condition $\operatorname{Tr} A=0$ is fulfilled, that is when

$$
F_{\mathrm{H}}=\frac{1}{2}\left[\sqrt{k}-2 k-\sqrt{(2 k-\sqrt{k})^{2}-4 k^{2}}\right] .
$$

In order for the system to present Turing structures when the space is taken into account, it must cross a Turing bifurcation point in the $(F, k)$ parameter space such that a stable steady state of the system becomes unstable to standing, space-periodic perturbations [Rovinsky and Menzinger 1992]. With the introduction of the diffusion terms, the nondiffusive stability conditions Eqs. (7)-(8) become

$$
\begin{gathered}
{\left[\sigma(F+k)-\left(v_{\mathrm{B}}^{2}+F\right)\right]^{2} / 4 \sigma>(F+k)\left(V_{\mathrm{B}}^{2}-F\right)} \\
\sigma(F+k)-\left(v_{\mathrm{B}}^{2}+F\right)>0
\end{gathered}
$$

with $\sigma \equiv D_{u} / D_{v}$, the diffusion coefficient ratio. Equations (7)-(8) and (10)-(11) are the conditions for diffusiondriven instability associated to the diffusive Gray-Scott model and define the domain in parameters' space $(F, k, \sigma)$ - called Turing space - for which spatial patterns grow (see Fig.1a). Mazin et al. [1996] delimitate this domain: the regions in the $(F, k)$ space where the blue state is unstable towards the growth of standing spatial perturbations, for several values of $\sigma$.

\section{Pattern Formation}

For the numerical study of the partial differential equations we used the conditions initially employed in Pearson [1993] and consisting in a system size of $R \times R$, with 
$R=2.5$ discretized through $x \rightarrow\left(x_{0}, x_{1}, x_{2}, \ldots, x_{N}\right)$ and $y \rightarrow\left(y_{0}, y_{1}, y_{2}, \ldots, y_{N}\right)$, with $N=256$. The numerical integration of the partial differential equations was performed by means of forward Euler integration, with a time step of $\tau \approx 0.9$ and spatial resolution $h=R / N$, and using the standard five-point approximation for the 2D Laplacian with periodic boundary conditions. More precisely, the concentrations $\left(u_{i, j}^{n+1}, v_{i, j}^{n+1}\right)$ at the moment $(n+1) \tau$ at the mesh position $(i, j)$ are given by

$$
\begin{aligned}
& u_{i, j}^{n+1}=u_{i, j}^{n}+\tau D_{u} \Delta_{h} u_{i, j}^{n}+\tau \mathcal{F}_{1}\left(u_{i, j}^{n}, v_{i, j}^{n}\right) \\
& v_{i, j}^{n+1}=v_{i, j}^{n}+\tau D_{v} \Delta_{h} v_{i, j}^{n}+\tau \mathcal{F}_{2}\left(u_{i, j}^{n}, v_{i, j}^{n}\right),
\end{aligned}
$$

with the Laplacian defined by

$$
\Delta_{h} u_{i, j}^{n}=\frac{u_{i+1, j}^{n}+u_{i, j+1}^{n}+u_{i-1, j}^{n}+u_{i, j-1}^{n}-4 u_{i, j}^{n}}{h^{2}} .
$$

The system was initialized with $\left(u_{\mathrm{i}}, v_{\mathrm{i}}\right)=(1,0)$ with the exception of a small central square of initial conditions $\left(u_{\mathrm{i}}, v_{\mathrm{i}}\right)=(0.5,0.25)$ perturbed with a $1 \%$ random noise. The works existent in the literature illustrate examples of patterns following a colormap on the U-concentration, with the red color representing the $\left(u_{\mathrm{R}}, v_{\mathrm{R}}\right)$ steady state and the blue one, a value in the vicinity of the $\left(u_{\mathrm{B}}, v_{\mathrm{B}}\right)$ state, e.g. $(u, v)=(0.3,0.25)$.

For an overview of the patterns resulting from Eqs. (3)(4), we have integrated the equations superimposing on the space $(X, Y) \equiv[0,2.5]^{2}$ a gradient of the control parameters, more precisely horizontally $k \in[0.03,0.07]$ and vertically $F \in[0,0.08]$. For a better resolution, we have used a $1024 \times 1024$ grid and the resultant "phase diagram" is shown in Fig.1a, where the bifurcation curves are also drawn. The two steady states from Eqs. (5)-(6) exist on the left of the saddle-node bifurcation curve, with the blue state being unstable about the growth of temporal fluctuations below the Hopf bifurcation curve. As the Hopf bifurcation is supercritical [see Rovinsky and Menzinger 1992] for $k<0.035$, the diffusionless model exhibits stable self-sustained oscillations only for these values of $k$, while for higher values of $k$, the subcritical nature of the bifurcation forces the system into expanding oscillations until it finally reaches the red state.

As discussed also in Pearson [1993], the spots occur only for the parameter values for which the only steady state is the red one - r.h.s. of the saddle-node bifurcation curve-, and thus the gradient needed for the formation and maintenance of the blue spots is an intrinsic self-sustaining feature of the system. Once a spot of high $V$ is formed, it is maintained by the concentration difference between its center and the surroundings of the spot. As the concentrations are limited to the $[0,1]$ interval, the spot can grow until the central concentration can no longer achieve the necessary gradient and thus its $V$-value starts to decrease. This induces the spot-division phase.

As examples of patterns implying spots multiplication, we have represented in Fig. $1 b, c, d$ also a selection of patterns sharing this property, while in panels e,f,g we illustrate a typical spot-replication process. In the Pearson terminology, the

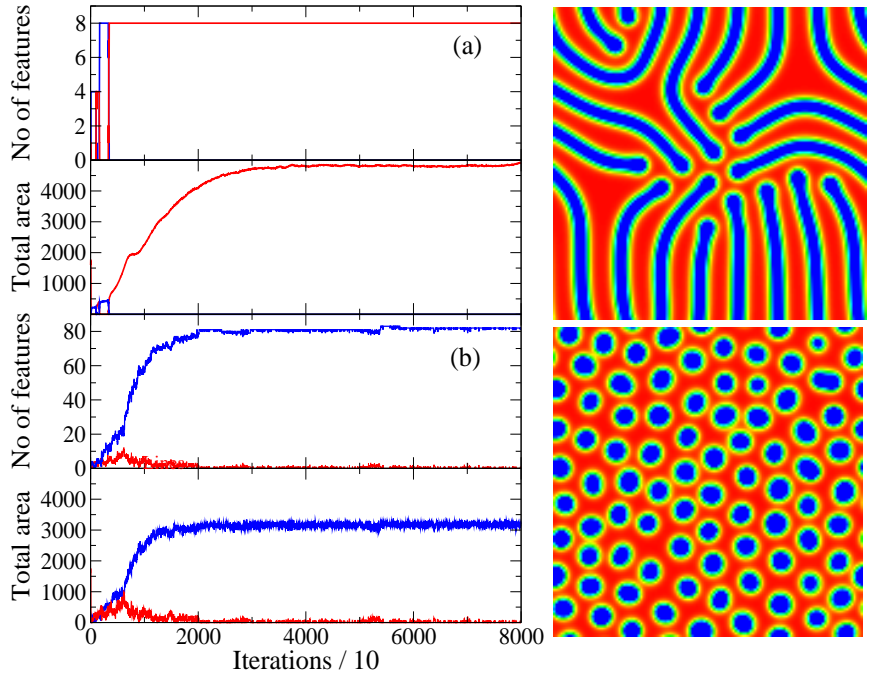

FIG. 2 The influence of noise on pattern formation for $(F, k)=$ (0.05, 0.0655) and: (a) $A=0.0$;(b) $A=0.0005$. Temporal evolution of the number of lines (red) and spots (blue) - upper panel - and of the total area encompassed by them - lower panels. R.h.s. panels show the spatial pattern obtained after 80000 iterations.

rightmost upper panels are an example of $\epsilon, \zeta$ and $\lambda$ patterns. For all of them, the process of spot multiplication continues until they fill the entire space. While the $\lambda$ pattern reaches a stationary state of completely packed spots, the other two patterns present a continuous formation and disappearance of spots, the latter being due to over-crowding and leading to the creation of extended red areas [Pearson 1993]. More precisely, the $\epsilon$ pattern is formed through the continuous disordered breaking of randomly spread filiform structures into spots, while for the $\zeta$ pattern, the area occupied by spots forms in the center and grows outward spherically.

\section{The Influence of Noise}

There is considerable interest in the scientific community for studying the implications of stochasticity in the evolution of biological and chemical systems. More precisely, often it has been speculated on the organizing and stabilizing role that noise might play in pattern formation, a somewhat counter-intuitive effect similar to the role played by diffusion in reaction-diffusion systems. Not being an exception, the Pearson model has been a target for such an investigation. Lesmes et al. [2003] have carried out the first study of the noise-controlled pattern formation in the Pearson model, with emphasis on the self-replicating patterns. They found that for the chosen value of the pair $(F, k)=(0.05,0.0655)$, the noise drives the system from the non-multiplicative, stripe-like pattern $(\kappa$ pattern $)$ to the spot-multiplication one ( $\lambda$ pattern $)-$ Fig2. The noise is included an additive Gaussian one with zero-mean and standard deviation controlled by the parameter $A$ as $\sigma^{2}=2 A$. Interesting enough, they argue in favor of an optimal noise intensity for which the number of spots is max- 

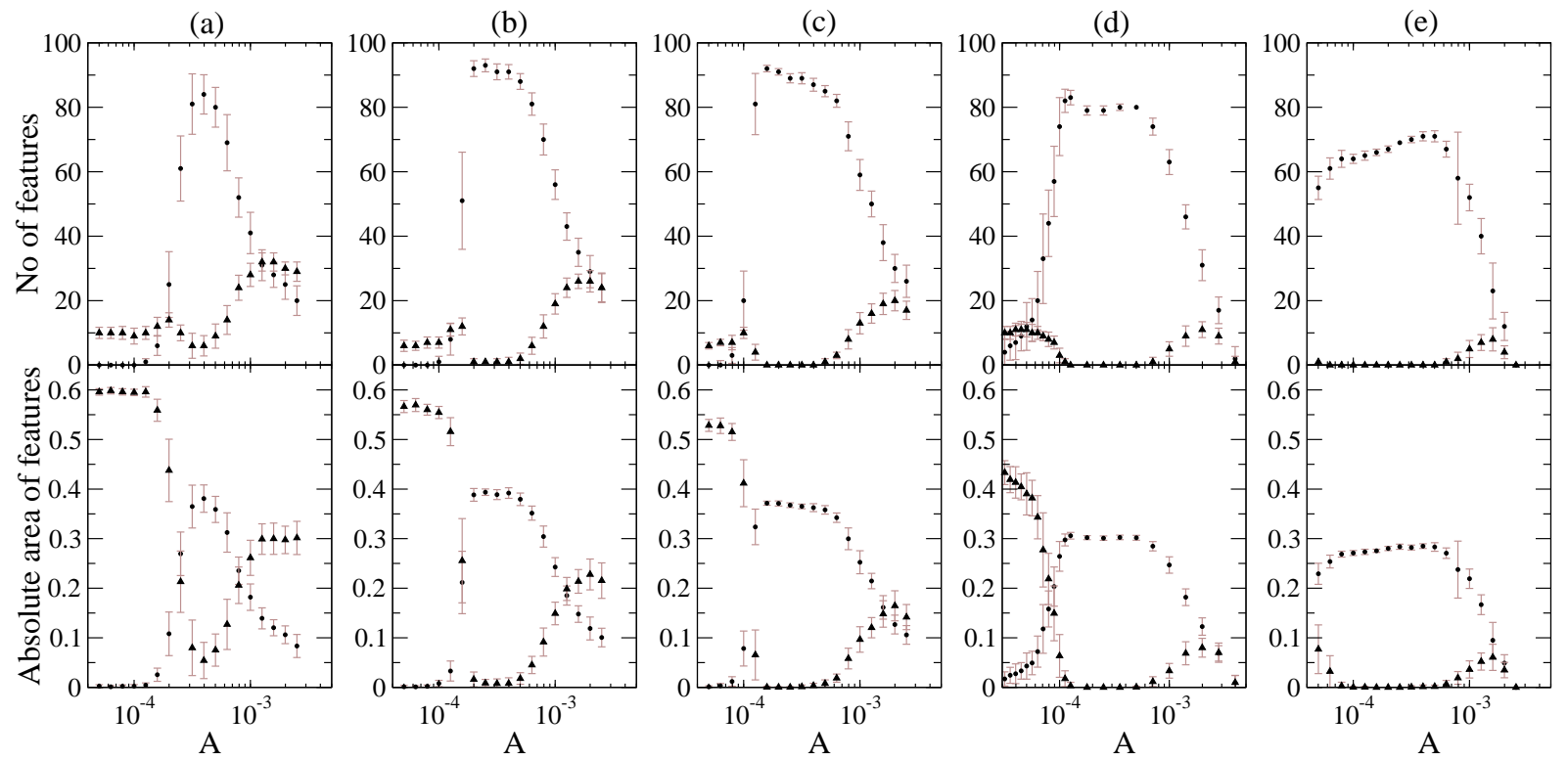

FIG. 3 The number of spots (upper panels - dots) and lines (upper panels - triangles) as a function of noise intensity. The area occupied by spots (lower panels - dots) and lines (lower panels - triangle) as a function of noise intensity. The parameters are $F=0.05$ and (a): $k=0.064,(b): k=0.0645,(c): k=0.065,(d): k=0.0655,(\mathrm{e}): k=0.066$.

imal, after a sufficiently long integration time. We reproduce their results in Fig.3(d) obtained from a statistics of 20 experiments and 100000 iterations for every $(F, k, A)$ chosen, for a spatial domain characterized by $R=1.25$ and $N=128$. Supplementary to their analysis, we included also the evolution of the number of lines/spots (upper panels) and the total normalized area occupied by spots and lines, respectively (lower panels). As suggested also by Fig.3, we consider the latter as a better tracer of the effect of noise on pattern formation. As component cells of a spot were considered those characterized by a value of $U<0.1$, while the total area was scaled to the real space by multiplying the number of spot's or line's cells with the scale factor $h^{2}$.

In comparison with the results of Lesmes et al., ours suggest the existence of an interval of optimum noise-intensity values leading to a maximum number of spots, rather than a single optimum value. In other words, our distribution of spots shows a plateau at the level of maximum number of spots, while theirs appears to have a more bell-like shape. The plateau characteristics is more evident in the distribution of the area occupied by the two features - spots and lines - as the noise intensity varies. In order to confirm this remark, we have carried out a similar analysis for various pairs of $(F, k)$, more precisely maintaining $F$ constant and varying the parameter $k$. The chosen $(F, k)$ pairs encompass several patterns, from completely stripe-like to completely spot-like patterns. One can see from Fig. 3 that in general there exists an optimumnoise interval that guarantees the maximum number of spots. When going to lower values of $k$ or equivalently deeper into the stripe-like pattern region, the noise loses its constructive role and the capability of pattern switching. Additionally, one can see that, once a certain noise threshold is reached, the transition into a spot-like dynamics is extremely fast compared with the slow decay of the number of spots when the noise level becomes destructive.

\section{ACKNOWLEDGMENTS}

One of us, A.M. thanks Pau Fernández for substantial assistance in the simulations of this model. This work has been supported by EU PACE grant within the 6th Framework Program under contract FP6-002035 (Programmable Artificial Cell Evolution), by MCyT grant FIS2004-05422 and by the Santa Fe Institute.

\section{References}

Gierer, A. and Meinhardt, H. [2000]. Theory of Biological Pattern Formation. Kybernetik, 12:30.

Gray, P. and Scott, S. K. [1985]. Sustained oscillations and other exotic patterns of behavior in isothermal reactions. J.Phys.Chem., 89:22.

Koch, A. J. and Meinhardt, H. [1994]. Biological pattern formation: from basic mechanisms to complex structures. Review of Modern Physics, 66:1481.

Lee, K. J., McCormick, W. D., Ouyang, Q., and Swinney, H. L. [1993]. Pattern formation by interacting chemical fronts. Science, 261:192.

Lee, K. J., McCormick, W. D., Swinney, H. L., and Pearson, J. E. [1994]. Experimental observation of self-replicating spots in a reaction-diffusion system. Nature, 369:215.

Lesmes, F., Hochberg, D., Moran, F., and Perez-Mercader, J. [2003]. Noise-controlled self-replicating patterns. Physical Review Letters, 91:238301. 
Mazin, W., Rasmussen, K. E., Mosekilde, E., Borckmans, P., and Dewel, G. [1996]. Pattern formation in the bistable Gray-Scott model. Mathematics and Computers in Simulation, 40:371.

Muratov, C. B. and Osipov, V. V. [2000]. Static spike autosolitons in the Gray-Scott model. J. Phys. A: Math. Gen., 33:8893.

Pearson, J. E. [1993]. Complex patterns in a simple system. Science, 261:189.

Rovinsky, A. and Menzinger, M. [1992]. Interaction of Turing and Hopf bifurcation in chemical systems. Phys. Rev. A, 46:6315.
Segel, L. A. and Jackson, J. L. [1972]. Dissipative structure: an explanation and an ecological example. J.Theor:Biol., 37:545.

Strogatz, S. [1994]. "Nonlinear Dynamics and Chaos with applications to Physics, Biology, Chemistry, and Engineering". Reading, MA: Addison-Wesley.

Turing, J. E. [1952]. The chemical basis of morphogenesis. Philos. Trans. Roy. Soc. London Ser. B, 327:37. 